\title{
LINEAR COMPLEMENTARITY AND ORIENTED MATROIDS
}

\author{
Komei Fukuda* \\ University of Tsukuba Delft University of Technology
}

(Received October 17, 1990; Revised September 27, 1991)

\begin{abstract}
A combinatorial abstraction of the linear complementarity theory in the setting of oriented matroids was first considered by M.J. Todd. In this paper, we take a fresh look at this abstraction, and attempt to give a simple treatment of the combinatorial theory of linear complementarity. We obtain new theorems, proofs and algorithms in oriented matroids whose specializations to the linear case are also new. For this, the notion of sufficiency of square matrices, introduced by Cottle, Pang and Venkateswaran, is extended to oriented matroids. Then, we prove a sort of duality theorem for oriented matroids, which roughly states: exactly one of the primal and the dual system has a complementary solution if the associated oriented matroid satisfies "weak" sufficiency. We give two different proofs for this theorem, an elementary inductive proof and an algorithmic proof using the criss-cross method which solves one of the primal or dual problem by using surprisingly simple pivot rules (without any perturbation of the original problem).
\end{abstract}

\section{Introduction}

The linear complementarity problem (LCP) is to find vectors $w$ and $z$ in $R^{n}$ satisfying

$$
\begin{aligned}
& w=A z+b, z \geq 0, w \geq 0 \\
& z^{T} w=0
\end{aligned}
$$

where $b$ is a given rational $n$-vector, and $A$ is a given rational $n \times n$ matrix.

The theory of linear complementarity, introduced by Cottle [7], Cottle and Dantzig [8] and Lemke [20], has been extensively studied for the last two decades. One main importance of this theory lies in the fact that it provides a unified framework for the two important optimization problems with wide applications, linear programming and convex quadratic programming.

It is known, however, that solving a general LCP (1.1) is hard: it is NP-complete, see [5]. The main difficulty lies in the fact that we don't know any easy way to recognize when the problem has no solution. For an LCP to be solved efficiently, it is necessary that a polynomially verifiable condition exists which is true if and only if the LCP has no solution. Such a condition may be called a good characterization of the nonexistence of solutions. There are several different classes of matrices $A$ for which such a characterization is known. The P-matrices and the positive semidefinite (PSD-) matrices constitute such classes. The LCPs arising from linear programming problems or convex quadratic programming problems have PSD-matrices. The class of sufficient matrices, which has been recently introduced by Cottle, Pang and Venkateswaran $[9,10]$, contains properly the two classes and admits also a good characterization of the nonexistence of solutions (The definition of sufficient matrices will be given in Section 2).

\footnotetext{
* Partially supported by RISE (Research Institute of Software Engineering Foundation, Japan). This paper was written while the first author was visiting INRIA and Université de Paris VI, France

${ }^{\dagger}$ Partially supported by OTKA No.2115 (Hungary) and RISE (Research Institute of Software Engineering Foundation, Japan)
} 
There are two major approaches in developing algorithms for such "good" classes of LCPs, the classical pivoting approach and the recent interior-point approach $[19,29]$. While polynomial-time algorithms for LCPs have been obtained only by the latter approach, a satisfactory foundation of linear complementarity theory depends strongly on the combinatorial nature of the former approach.

Todd $[26,27]$ was the first to explain the essence of combinatorics used in the classical pivoting algorithms for LCPs and extend many fundamental results on LCP to the notion of oriented matroids. In order to achieve these he obtained elegant characterizations of symmetric matrices, P-matrices and PSD-matrices in terms of sign conditions on the linear subspaces associated with the extended matrix $[-A I]$. Another difficulty he had to overcome was to generalize perturbation techniques to oriented matroids, since the usual pivoting algorithms for LCP rely heavily on nondegeneracy.

On the other hand, the recent studies by Klafszky and Terlaky [17, 18], Fukuda and Terlaky [15] and den Hertog, Roos and Terlaky [11] showed that the nondegeneracy assumption is not necessary for the finiteness of pivoting algorithm if the criss-cross type of "minimum-index" pivot strategy is employed.

In this paper, we attempt to do two things. The first thing is to obtain a general theorem, called the duality theorem, of complementarity in the setting of oriented matroids, which gives as a corollary a good characterization of the nonexistence of a solution for LCPs with sufficient matrices. For this, a certain combinatorial abstraction of sufficient matrices is useful for the simplicity of the statement and also for a short elementary proof. The second thing is to study essential conditions on oriented matroids under which the crisscross method [18], a natural extension of the criss-cross method for linear programming $[24,25,28]$ solves the associated complementarity problem. For this purpose, we extract the combinatorial ideas used in [11]. It turns out that if an oriented matroid satisfies essentially the same assumption as for the duality theorem, the criss-cross method solves the complementarity problem, and hence gives a constructive proof of the duality theorem. It is noteworthy that we do not depend on the nondegeneracy assumption. And therefore, we not only generalize some theorems of Cottle et al [9] and Todd [26, 27], but simplify their proofs, and obtain a new algorithm which is extremely simple.

\section{LCP Duality Theorem}

In order to write the LCP (1.1) in a form that will be convenient for extending it to the setting of oriented matroids, we set

$$
V(A, b)=\left\{x \in R^{2 n+1} \mid[-b-A I] x=0\right\} .
$$

We denote the components of a vector $x \in R^{2 n+1}$ by $x=\left(x_{0}, x_{1}, x_{2}, \ldots, x_{2 n}\right)$ instead of the usual $x=\left(x_{1}, x_{2}, \ldots, x_{2 n+1}\right)$, while we denote a vector $x \in R^{2 n}$ by $x=\left(x_{1}, \ldots, x_{2 n}\right)$. Then, one can rewrite the LCP (1.1) as

$$
\begin{aligned}
& \text { find } x \in V(A, b) \text { satisfying } \\
& x \geq 0, x_{0}=1 \\
& x_{i} \cdot x_{n+i}=0 \text { for all } i=1, \ldots, n .
\end{aligned}
$$

A vector $x$ in $R^{2 n}$ or $R^{2 n+1}$ is called complementary if the complementarity condition (2.4) is satisfied. A solution to the LCP is a nonnegative complementary vector $x$ in $V(A, b)$ with $x_{0}=1$. 
If the LCP has a solution, then it is easy to see that there is a basic solution, i.e., one having a minimal support among all nonzero vectors in $V(A, b)$, and hence the LCP is in $N P$ (see [23], Corollary 3.2b). In general, there is no known good characterization of the nonexistence of a solution to the LCP. However we can write conditions on the linear subspace under which such characterization exists.

A vector $x$ in $R^{2 n}$ or in $R^{2 n+1}$ is called strictly sign-preserving (s.s.p.) if

$$
\begin{aligned}
& x_{i} \cdot x_{n+i} \geq 0 \text { for all } i=1, \ldots, n \text { and } \\
& x_{j} \cdot x_{n+j}>0 \text { for some } j
\end{aligned}
$$

and called strictly sign-reversing (s.s.r.) if

$$
\begin{aligned}
& x_{i} \cdot x_{n+i} \leq 0 \text { for all } i=1, \ldots, n \text { and } \\
& x_{j} \cdot x_{n+j}<0 \text { for some } j .
\end{aligned}
$$

Now we have the following general theorem, which we call the duality theorem of linear complementarity.

Theorem 2.1 (LCP Duality Theorem) Let $V$ be a linear subspace of $R^{2 n+1}$ satisfying the following condition:

$$
\begin{aligned}
\text { either } & V \text { contains no s.s.r. vector } x \text { with } x_{0}=0 \\
\text { or } & V \text { contains no s.s.p. vector } x \text { with } x_{0} \neq 0 \text {, }
\end{aligned}
$$

and suppose the orthogonal complement $V^{\perp}$ also satisfies the same condition, i.e.,

$$
\begin{aligned}
\text { either } & V^{\perp} \text { contains no s.s.r. vector } y \text { with } y_{0}=0 \\
\text { or } & V^{\perp} \text { contains no s.s.p. vector } y \text { with } y_{0} \neq 0 .
\end{aligned}
$$

Then exactly one of the following statements holds:

(a) there exists a nonnegative complementary vector $x$ in $V$ with $x_{0}=1$;

(b) there exists a nonnegative complementary vector $y$ in $V^{\perp}$ with $y_{0}=1$.

One can easily see that this theorem is self-dual, i.e., replacing $V$ by $V^{\perp}$ yields the same theorem. This self-duality makes the statement look simple, and quite importantly it makes a short elementary proof possible. Also, this theorem suggests us to consider the dual problem of the LCP $(2.2) \sim(2.4)$ :

$$
\begin{aligned}
& \text { find } y \in V(A, b)^{\perp} \text { satisfying } \\
& y \geq 0, y_{0}=1 \\
& y_{i} \cdot y_{n+i}=0 \text { for all } i=1, \ldots, n,
\end{aligned}
$$

which is solvable if and only if the primal problem is not, under the assumptions of theorem. Remark that if $V$ in the duality theorem has an explicit representation as $V(A, b)$, then one can write the statements (a) and (b) more explicitly as

(a') there exist vectors $w, z$ in $R^{n}$ satisfying $w=A z+b, z \geq 0, w \geq 0$, and $z^{T} w=0$;

(b') there exists a vector $u$ in $R^{n}$ satisfying $b^{T} u=-1, u \geq 0, A^{T} u \leq 0$, and $u^{T} A^{T} u=0$. 
The assumptions (2.9) and (2.10) for $V(A, b)$ are satisfied by several well-known classes. of square matrices $A$, for any vector $b$. For example, the class of $\mathrm{P}$-matrices and the class of PSD-matrices satisfy the conditions because, for any $b$,

$$
\begin{aligned}
& V(A, b) \text { contains no s.s.r. vector } x \text { with } x_{0}=0 \text { and } \\
& V(A, b)^{\perp} \text { contains no s.s.p. vector } w \text { with } w_{0} \neq 0 .
\end{aligned}
$$

In fact, the sufficient matrices are exactly those matrices with these properties. More explicitly, an $n \times n$ matrix $A$ is called column sufficient if

$$
w=A z \text { implies }(w, z) \text { is not s.s.r., }
$$

called row sufficient if

$$
v=-A^{T} u \text { implies }(u, v) \text { is not s.s.p., }
$$

and called sufficient if it is both column and row sufficient. Clearly, the row-sufficiency of $A$ is equivalent to the column-sufficiency of $A^{T}$. Theorem 2.1 implies that for any sufficient matrix $A$ and any vector $b$, exactly one of statements (a') or (b') holds.

As an example of linear subspaces $V$ satisfying the conditions (2.9) and (2.10), we can set $A=\left[\begin{array}{ll}0 & 1 \\ 0 & 1\end{array}\right], b=\left[\begin{array}{l}-1 \\ -1\end{array}\right]$, and $V=V(A, b)$. In this example, the primal problem has a complementary solution $x=\left(x_{0}, x_{1}, x_{2}, x_{3}, x_{4}\right)=(1,0,1,0,0)$. Remark that if we set $b=\left[\begin{array}{l}-1 \\ -\frac{1}{2}\end{array}\right]$ and use the same $A$, the conditions of the theorem are not fulfilled because both the s.s.r vector $(0,0,1,1,-2)$ and the s.s.p. vector $(2,0,-1,3,-2)$ are in $V^{\perp}$. In fact, in this case, neither of $V$ nor $V^{\perp}$ have any nonnegative complementary solutions, although $V$ contains the nonnegative vector $\left(1,0,1,0, \frac{1}{2}\right)$. The matrix $A$ is column sufficient (and not row sufficient), and this example explains the difficulty of solving the LCP with a column sufficient matrix in general.

It should be noted that Theorem 2.1 can be considered as a generalization of the wellknown LP duality theorem and Cottle's QP duality theorem [6] since they correspond to certain subclasses of PSD-matrices. Readers interested in these relations are suggested to see [27] for reformulations of the LP and QP duality theorems in the complementarity framework.

In the following section, we will see how the duality theorem, Theorem 2.1 , can be generalized and proved in purely combinatorial fashion using the notion of oriented matroids.

\section{Oriented Matroids and Complementarity}

Let $E$ be a finite set. A signed vector $X$ on $E$ is a vector in $\{+, 0,-\}^{E}$. The opposite $-X$ of a signed vector $X$ is defined in the obvious way: $-(-0-++)=(+0+--)$. Let $X$ and $Y$ be signed vectors on $E$. An element $j \in E$ is said to separate $X$ and $Y$ if $X_{j}$ and $Y_{j}$ are nonzero and opposite in signs. The set of elements separating $X$ and $Y$ is denoted by $D(X, Y)$. The composition of $X$ and $Y$, denoted by $X \circ Y$, is the signed vector on $E$ defined by

$$
(X \circ Y)_{j}=\left\{\begin{array}{ll}
X_{j} & \text { if } X_{j} \neq 0 \\
Y_{j} & \text { otherwise }
\end{array} \quad(j \in E) .\right.
$$

The support $\underline{X}$ of $X$ is the set $\left\{j \in E: X_{j} \neq 0\right\}$. 
An oriented matroid on $E$ is a pair $M=(E, \mathcal{F})$, where $E$ is a finite set and $\mathcal{F}$ is a set of signed vectors on $E$ satisfying the following axioms:

(OM1) $\quad \mathbf{0} \in \mathcal{F} ; \quad$ (where $\mathbf{0}$ denotes the zero vector)

(OM2) $X \in \mathcal{F} \Longrightarrow-X \in \mathcal{F}$;

(OM3) $X, X^{\prime} \in \mathcal{F} \Longrightarrow X \circ X^{\prime} \in \mathcal{F}$

(OM4) $X, X^{\prime} \in \mathcal{F}$ and $f \in D\left(X, X^{\prime}\right) \Longrightarrow$ there exists $Z \in \mathcal{F}$ such that $Z_{f}=0$ and $Z_{j}=\left(X \circ X^{\prime}\right)_{j}$ for all $j \notin D\left(X, X^{\prime}\right)$.

We call each member of $\mathcal{F}$ a vector of the oriented matroid. Each vector with minimal nonempty support is called a circuit. One can define oriented matroids in terms of circuits, see $[2,3]$.

Note that the notion of oriented matroids was introduced independently by Bland-Las Vergnas [3] and Folkman-Lawrence [12]. The definition of oriented matroids above is due to Edmonds, Fukuda and Mandel [13, 21].

For a vector $x \in R^{E}$, we denote the signed incidence vector of $x$ as $\delta(x)$, i.e., $\delta(x)$ is the signed vector on $E$ with $\delta(x)_{j}=\operatorname{sign}\left(x_{j}\right)$ for each $j \in E$. One can easily check that for any linear subspace $V$ of $R^{E}$, the set $\mathcal{F}=\delta(V)$ of all signed vectors coming from $V$ satisfies the axioms $(\mathrm{OM} 1) \sim(\mathrm{OM} 4)$. Such an oriented matroid is called representable or linear. There are several interesting constructions of non-representable oriented matroids, see $[13,21,4,1]$.

We shall give below some basic properties of oriented matoids without proofs. For those unfamiliar with oriented matroids, good references are $[1,3,12,13,21]$.

It will be convenient to define the product $\alpha \cdot \beta$ (or simply $\alpha \beta$ ) of two signs $\alpha, \beta \in$ $\{+, 0,-\}$ in the natural way: + if both are nonzero and of same sign, - if both are nonzeroand of opposite sign, and 0 otherwise. Two signed vectors $X$ and $Y$ are called orthogonal (denoted by $X * Y$ ) if the existence of an element $f \in E$ with $X_{f}, Y_{f} \neq 0$ implies the existence of an element $g \in E$ with $X_{g} \cdot Y_{g}=-X_{f} \cdot Y_{f}$. The dual $M^{*}=\left(E, \mathcal{F}^{*}\right)$ of an oriented matroid $M$ is defined by

$$
\mathcal{F}^{*}=\left\{Y \in\{+,-, 0\}^{E} \mid X * Y \text { for all } X \in \mathcal{F}\right\} .
$$

The dual $M^{*}$ is also an oriented matroid, and

$$
\mathcal{F}^{* *}=\mathcal{F}
$$

Furthermore, if $F=\delta(V)$ for a linear subspace $V$ of $R^{E}$ then $F^{*}=\delta\left(V^{\perp}\right)$. A vector, circuit, ... of $M^{*}$ may be called covector, cocircuit ... of $M$.

There are two basic operations on oriented matroids which will be used frequently in this paper. For a subset $S$ of $E$, let

$$
\begin{aligned}
& \mathcal{F} \backslash S=\left\{X \backslash S \mid X \in \mathcal{F} \text { and } X_{j}=0 \text { for all } j \in S\right\} \\
& \mathcal{F} / S=\{X \backslash S \mid X \in \mathcal{F}\},
\end{aligned}
$$

where $X \backslash S$ denotes the subvector $\left(X_{j}: j \in E \backslash S\right)$ of $X$. It is easy to see from the definition, both $(E, \mathcal{F} \backslash S)$ and $(E, \mathcal{F} / S)$ are oriented matroids, which are denoted by $M \backslash S$ and $M / S$, respectively. When $S$ is a singleton set $\{e\}, M \backslash\{e\}$ and $M /\{e\}$ will be denoted by $M \backslash e$ and $M / e$, respectively. The following relations hold for any oriented matroid $M=(E, \mathcal{F})$ :

$$
\begin{aligned}
& (\mathcal{F} \backslash S)^{*}=\mathcal{F}^{*} / S \\
& (\mathcal{F} / S)^{*}=\mathcal{F}^{*} \backslash S
\end{aligned}
$$


For a nonnegative integer $n$, let $E_{2 n}$ denote a set of $2 n$ elements partitioned into prescribed $n$ pairs; for each $e \in E_{2 n}$ there is a unique element $\bar{e}$ in $E_{2 n}$, called the complement of $e$ such that $\bar{e} \neq e$ and $\overline{\bar{e}}=e$. For a subset $S$ of $E_{2 n}$ let $\bar{S}=\{\bar{e} \mid e \in S\}$. A subset $S$ is called complementary if $S \cap \bar{S}=\emptyset$. We use $\hat{E}_{2 n}$ to denote $E_{2 n} \cup\{g\}$ for a given $E_{2 n}$ and a distinguished element $g$ which is assumed to be not in $E_{2 n}$.

One canonical choice of $E_{2 n}$ and $\hat{E}_{2 n}$ is $\{1,2, \ldots, 2 n\}$ and $\{0,1,2, \ldots, 2 n\}$ with $g=0$, respectively, where $\bar{i}=i+n$ if $1 \leq i \leq n$, and $\bar{i}=i-n$ if $n<i \leq 2 n$.

For a given oriented matroid $M=\left(\hat{E}_{2 n}, \mathcal{F}\right)$ with $n \geq 0$, the complementarity problem $(\mathrm{OMCP})$ is to

$$
\begin{aligned}
& \text { find } X \in \mathcal{F} \text { satisfying } \\
& X \geq 0, X_{g}=+ \\
& X_{e} \cdot X_{\bar{e}}=0 \text { for all } e \in E_{2 n}
\end{aligned}
$$

Here, we use the inequality $\geq$ in the obvious way and we shall do so in the sequel. The conditions (3.6), (3.7) and (3.8) are straightforward abstractions of (2.2), (2.3) and (2.4), respectively.

Again, we simply make the "automatic" abstraction of definitions in the LCP case. A signed vector $X$ on $E_{2 n}$ or on $\hat{E}_{2 n}$ is called complementary if the condition (3.8) is satisfied, called strictly sign-preserving (s.s.p.) if

$$
\begin{aligned}
& X_{e} \cdot X_{\bar{e}} \geq 0 \text { for all } e \in E_{2 n} \text { and } \\
& X_{f} \cdot X_{\bar{f}}>0 \text { for some } f,
\end{aligned}
$$

and called strictly sign-reversing (s.s.r.) if

$$
\begin{aligned}
& X_{e} \cdot X_{\bar{e}} \leq 0 \text { for all } e \in E_{2 n} \text { and } \\
& X_{f} \cdot X_{\bar{f}}<0 \text { for some } f .
\end{aligned}
$$

The following theorem is a combinatorial generalization of the duality theorem, Theorem 2.1 .

Theorem 3.1 (OMCP Duality Theorem) Let $M=\left(\hat{E}_{2 n}, \mathcal{F}\right)$ be an oriented matroid satisfying the following conditions:

$$
\begin{aligned}
\text { either } & \mathcal{F} \text { contains no s.s.r. vector } X \text { with } X_{g}=0 \\
\text { or } & \mathcal{F} \text { contains no s.s.p. vector } X \text { with } X_{g} \neq 0 \text { and, } \\
\text { either } & \mathcal{F}^{*} \text { contains no s.s.r. vector } Y \text { with } Y_{g}=0 \\
\text { or } & \mathcal{F}^{*} \text { contains no s.s.p. vector } Y \text { with } Y_{g} \neq 0 .
\end{aligned}
$$

Then exactly one of the following statements holds:

(a) there exists a nonnegative complementary vector $X$ in $\mathcal{F}$ with $X_{g}=+$;

(b) there exists a nonnegative complementary vector $Y$ in $\mathcal{F}^{*}$ with $Y_{g}=+$.

We call an oriented matroid $M^{\prime}=\left(E_{2 n}, \mathcal{F}^{\prime}\right)$ sufficient if $\mathcal{F}^{\prime}$ contains no s.s.r. vector and its dual $\left(\mathcal{F}^{\prime}\right)^{*}$ contains no s.s.p. vector. If $M=\left(\hat{E}_{2 n}, \mathcal{F}\right)$ is an single-element extension of a sufficient oriented matroid $M^{\prime}=\left(E_{2 n}, \mathcal{F}^{\prime}\right)$ by $g$, i.e., $M \backslash g=M^{\prime}$, then clearly $M$ satisfies the conditions (3.13) and (3.14) of Theorem 3.1. Therefore we have the following corollary of the duality theorem. 
Corollary 3.2 Let $M=\left(\hat{E}_{2 n}, \mathcal{F}\right)$ be any single-element extension of a sufficient oriented matroid $M^{\prime}$ by $g$. Then exactly one of the following statements holds:

(a) there exists a nonnegative complementary vector $X$ in $\mathcal{F}$ with $X_{g}=+$;

(b) there exists a nonnegative complementary vector $Y$ in $\mathcal{F}^{*}$ with $Y_{g}=+$.

In this section, we prove the duality theorem in a most elementary way. It will be interesting to compare this proof with an algorithmic proof for (a slightly different version of) this theorem, since they are closely related.

For the elementary proof, what we need is the notion of minors of OMCP, which was introduced by Todd [26]. Let $M=\left(\hat{E}_{2 n}, \mathcal{F}\right)$ be an oriented matroid with $n \geq 0$. For each complementary subset $S$ of $E_{2 n}$, let $\left.M(S)=\left(\hat{E}_{2 n} \backslash S \cup \bar{S}\right), \mathcal{F}(S)\right)$ denote the oriented matroid $M \backslash S / \bar{S}$, which is called a complementary minor of $M$. For $e \in E$, we denote $M(\{e\})$ by $M(e)$. Of course, the set $\hat{E}_{2 n} \backslash(S \cup \bar{S})$ can be considered as a set of form $\hat{E}_{2(n-s)}$, for any complementary subset $S$ with $|S|=s$. From the definitions, we have the following relations:

$$
\begin{aligned}
& X \in \mathcal{F} \text { and } X_{e}=0 \text { for all } e \in S \Rightarrow X \backslash(S \cup \bar{S}) \in \mathcal{F}(S) ; \\
& X^{\prime} \in \mathcal{F}(S) \Rightarrow \exists X \in \mathcal{F} \text { such that } X \backslash(S \cup \bar{S})=X^{\prime} \text { and } X_{e}=0 \forall e \in S ; \\
& Y \in \mathcal{F}^{*} \text { and } Y_{\bar{e}}=0 \text { for all } e \in S \Rightarrow Y \backslash(S \cup \bar{S}) \in \mathcal{F}(S)^{*} ; \\
& Y^{\prime} \in \mathcal{F}(S)^{*} \Rightarrow \exists Y \in \mathcal{F}^{*} \text { such that } Y \backslash(S \cup \bar{S})=Y^{\prime} \text { and } Y_{\bar{e}}=0 \forall e \in S .
\end{aligned}
$$

These immediately imply the following:

Lemma 3.3 Let $M=\left(\hat{E}_{2 n}, \mathcal{F}\right)$ be an oriented matroid satisfying the conditions (3.13) and (3.14) of Theorem 3.1. Then the complementary minor $M(S)$ of $M$ satisfies the same conditions for any complementary subset $S$ of $E_{2 n}$.

Lemma 3.4 Let $M=\left(\hat{E}_{2 n}, \mathcal{F}\right)$ be an oriented matroid satisfying the conditions (3.13) and (3.14) of Theorem 3.1. Then for each $e \in E_{2 n}$, at most one of the following four statements holds:

(a1) $\exists$ a complementary vector $X^{1}$ in $\mathcal{F}$ with $X^{1} \backslash\{e, \bar{e}\} \geq 0, X_{\bar{e}}^{1}=-$ and $X_{g}^{1}=+$;

(a2) $\exists$ a complementary vector $X^{2}$ in $\mathcal{F}$ with $X^{2} \backslash\{e, \bar{e}\} \geq 0, X_{e}^{2}=-$ and $X_{g}^{2}=+$;

(b1) $\exists$ a complementary vector $Y^{1}$ in $\mathcal{F}^{*}$ with $Y^{1} \backslash\{e, \bar{e}\} \geq 0, Y_{e}^{1}=-$ and $Y_{g}^{1}=+$;

(b2) $\exists$ a complementary vector $Y^{2}$ in $\mathcal{F}^{*}$ with $Y^{2} \backslash\{e, \bar{e}\} \geq 0, Y_{\bar{e}}^{2}=-$ and $Y_{g}^{2}=+$.

Proof. We prove by contradiction. Suppose the conclusion is false, i.e., two of the four statements hold. We have six cases to consider but because of the duality (3.1), (3.4) and (3.5), we have essentially three cases:

Case 1. (a1) and (a2) hold. (This covers the case (b1) and (b2), also.)

Let $Z^{\prime}=X^{1} \circ X^{2}$. By the axiom (OM3), $Z^{\prime} \in \mathcal{F}$. It is easy to see from (a1) and (a2) that $Z^{\prime}$ is s.s.p. and $Z_{g}^{\prime} \neq 0$. On the other hand, by using the axiom (OM4) with $X=X^{1}$, $X^{\prime}=-X^{2}$ and $f=g$, we have $Z^{\prime \prime} \in \mathcal{F}$ such that $Z_{g}^{\prime \prime}=0$ and $Z_{j}^{\prime \prime}=\left(X^{1} \circ-X^{2}\right)_{j}$ for all $j \notin D\left(X^{1},-X^{2}\right)$. Again using (a1) and (a2), we have $Z_{j}^{\prime \prime} \cdot Z_{j}^{\prime \prime} \leq 0$ for all $j \in E_{2 n}$ and $Z_{e}^{\prime \prime} \cdot Z_{\bar{e}}^{\prime \prime}<0$. This means that $Z^{\prime \prime}$ is s.s.r. and $Z_{g}^{\prime \prime}=0$. The existence of $Z^{\prime}$ and $Z^{\prime \prime}$ violates the condition (3.13). Thus (a.1) and (a2) cannot hold simultaneously. 
Case 2. (a1) and (b1) hold. (This covers the case (a2) and (b2), also.)

Clearly, the vectors $X^{1}$ and $Y^{1}$ are not orthogonal, a contradiction. Thus (a1) and (b1) cannot hold simultaneously.

Case 3. (a1) and (b2) hold. (This covers the case (a2) and (b1), also.)

Clearly, the vectors $X^{1}$ and $Y^{2}$ are not orthogonal, a contradiction. Thus (a1) and (b2) cannot hold simultaneously.

This completes the proof.

Now we are ready to prove the duality theorem.

Proof of Theorem 3.1. We use induction on $n$. If $n=0$ then the theorem is obvious. Let $n>0$, and suppose that the theorem is true for all smaller values of $n$. Assume the assumptions (3.13) and (3.14) are satisfied. Let $e$ be an element of $E_{2 n}$. By Lemma 3.3, the assumptions of the theorem are satisfied by the minors $M(e)$ and $M(\bar{e})$. By inductive hypothesis, exactly one of

(a1') there exists a nonnegative complementary vector $\hat{X}^{1}$ in $\mathcal{F}(e)$ with $\hat{X}_{g}^{1}=+$;

(b1') there exists a nonnegative complementary vector $\hat{Y}^{1}$ in $\mathcal{F}(e)^{*}$ with $\hat{Y}_{g}^{-1}=+$ holds, and exactly one of

$\left(\mathbf{a} 2^{\prime}\right)$ there exists a nonnegative complementary vector $\hat{X}^{2}$ in $\mathcal{F}(\bar{e})$ with $\hat{X}_{g}^{2}=+$;

(b2') there exists a nonnegative complementary vector $\hat{Y}^{2}$ in $\mathcal{F}(\bar{e})^{*}$ with $\hat{Y}_{g}^{2}=+$

holds. Now assume that the conclusion of the theorem is false, i.e., neither (a) nor (b) of the theorem holds. Then, using the relations (3.16) and (3.18), each of the statements (a1'), $\left(\mathrm{a} 2^{\prime}\right),\left(\mathrm{b} 1^{\prime}\right)$ and (b2') implies

(a1) $\exists$ a complementary vector $X^{1}$ in $\mathcal{F}$ with $X^{1} \backslash\{e, \bar{e}\} \geq 0, X_{\bar{e}}^{1}=-$ and $X_{g}^{1}=+$;

(a2) $\exists$ a complementary vector $X^{2}$ in $\mathcal{F}$ with $X^{2} \backslash\{e, \bar{e}\} \geq 0, X_{e}^{2}=-$ and $X_{g}^{2}=+$;

(b1) $\exists$ a complementary vector $Y^{1}$ in $\mathcal{F}^{*}$ with $Y^{1} \backslash\{e, \bar{e}\} \geq 0, Y_{e}^{1}=-$ and $Y_{g}^{1}=+$;

(b2) $\exists$ a complementary vector $Y^{2}$ in $\mathcal{F}^{*}$ with $Y^{2} \backslash\{e, \bar{e}\} \geq 0, Y_{\bar{e}}^{2}=-$ and $Y_{g}^{2}=+$,

respectively. The induction hypothesis forces that two of the statements (a1), (a2), (b1) and (b2) must hold. But this is impossible from Lemma 3.4. Thus the one of two statements (a) and (b) must hold. It is clear that (a) and (b) cannot hold simultaneously, and the theorem follows.

\section{Basis, Tableau and the Duality Theorem}

In the previous section, we have proved the duality theorem by an elementary inductive argument. The proof was very simple and, in fact, it gives some general idea of how the OMCP can be solved; once you solve two subproblems $M(e)$ and $M(\bar{e})$, then one of the two solutions will yield a solution to the original OMCP.

In this section, we will see how this simple idea can be extended to a finite algorithm. For this, we need the notion of basis, as this is essential in pivoting algorithms for the linear complementarity problem. 
Let $M=(E, \mathcal{F})$ be an oriented matroid. A basis is a maximal subset $B$ of $E$ such that there is no nonzero vector $X$ in $\mathcal{F}$ with $\underline{X} \subseteq B$. The following properties of basis are well-known:

Property 4.1 Let $M=(E, \mathcal{F})$ be an oriented matroid.

(1) Every basis has the same cardinality, called the $\operatorname{rank} r(M)$ of $M$;

(2) For each basis $B$ and for each $j \in E \backslash B$, there exist a unique circuit, called the fundamental circuit $X(B, j)=X_{M}(B, j)$, such that $X(B, j)_{j}=+$ and $X(B, j) \subseteq B \cup\{j\}$;

(3) A subset $B$ of $E$ is a basis of $M$ if and only if $E \backslash B$ is a cobasis, i.e., a basis of the dual $M^{*}$.

We will usually denote by $N$ the cobasis $E \backslash B$, and denote by $Y(N, i)$ the fundamental cocircuit $X_{M^{*}}(N, i)$ for each $i \in B$

For each basis $B$ and the cobasis $N=E \backslash B$, the tableau $T(B)=\left(t_{i j}: i \in B, j \in N\right)$ is the $\{+, 0,-\}$-matrix defined by

$$
t_{i j}=X(B, j)_{i} \text { for } i \in B, j \in N \text {. }
$$

Clearly, the tableau $T(B)$ contains not only all information about the fundamental circuits $X(B, j), j \in N$, but also contains that of the fundamental cocircuits $Y(N, i), i \in B$ through the following well-known relationship:

$$
t_{i j}=-Y(N, i)_{j} \text { for } i \in B, j \in N \text {. }
$$

Therefore, the row vectors $\left(t_{i j}: j \in N\right), i \in B$ represent the essential part of the fundamental cocircuits with opposite sign, while the column vectors $\left(t_{i j}: i \in B\right), j \in N$ represent that of the fundamental circuits with same sign.

The following property is well-known and can be easily verified from the oriented matroid axioms $(\mathrm{OM} 1) \sim(\mathrm{OM} 4)$ and the definitions.

Lemma 4.2 Let $M=(E, \mathcal{F})$ be an oriented matroid. Let $B$ be a basis, and let $t_{r s}, r \in$ $B, s \in N$ be any nonzero entry. Then the set $B^{\prime}=B \backslash\{r\} \cup\{s\}$ is a basis, and the tableaux $T(B)=\left(t_{i j}\right)$ and $T\left(B^{\prime}\right)=\left(t_{i j}^{\prime}\right)$ are related as follows: for each $i \in B \backslash\{r\}$ and $j \in N \backslash\{s\}$,

$$
\begin{aligned}
t_{s r}^{\prime} & =t_{r s} \\
t_{s j}^{\prime} & =-t_{r s} \cdot t_{r j} \\
t_{i r}^{\prime} & =t_{r s} \cdot t_{i s}, \text { and } \\
t_{i j}^{\prime} & =t_{i j} \circ\left(-t_{r \cdot s} \cdot t_{i s} \cdot t_{r \cdot j}\right) \quad \text { unless } t_{i j}=t_{r \cdot s} \cdot t_{i s} \cdot t_{r j} \neq 0 .
\end{aligned}
$$

The replacement of a tableau $T(B)$ by an "adjacent" tableau $T\left(B^{\prime}\right)$ is called a pivot operation on the position $(r, s)$.

Now we come back to the complementarity problem. Let $M=\left(\hat{E}_{2 n}, \mathcal{F}\right)$ be an oriented matroid with $n \geq 0$. For the complementarity problems for which we give an algorithm, we assume that

Assumption 4.3 $M=\left(\hat{E}_{2 n}, \mathcal{F}\right)$ contains a basis $B$ with $|B|=n, g \notin B$ and complementary, i.e., $B \cap \bar{B}=\emptyset$. 

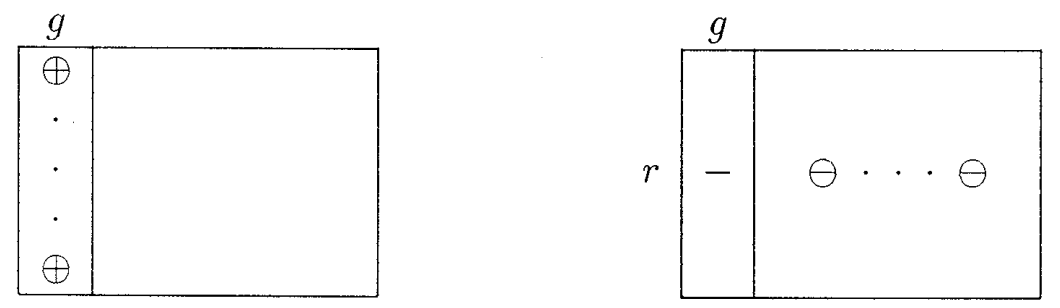

Figure 4.1: feasible and cofeasible tableaux

This assumption is always satisfied for the case of LCP (1.1) where $\mathcal{F}$ arises from the linear subspace $V(A, b)$ in $(2.1)$ the set $\{n+1, n+2, \ldots, 2 n\}$ is a complementary basis.

We define here two types of basis. A basis $B$ or tableau $T(B)$ is called feasible if $g \notin B$ and $X(B, g) \geq 0$, and called cofeasible if $g \notin B$, there exists $r \in B$ such that $t_{r g}<0$, and $t_{r j} \leq 0$ for all $j \in N$, where $N=\hat{E}_{2 n} \backslash B$. (See Figure 4.1 where the symbols $\oplus$ and $\ominus$ indicate a nonnegative entry and a nonpositive entry.)

Property 4.4 Let $B$ be a basis with $g \notin B$, and let $N$ be the cobasis $\hat{E}_{2 n} \backslash B$.

(1) If $B$ is feasible, then $X(B, g)$ is feasible, and if in addition complementary then $X(B, g)$ is complementary as well and solves the $O M C P$.

(2) If $B$ is cofeasible, then $Y(N, r)$ is cofeasible, and if in addition $B$ is complementary and $Y(N, r)_{\bar{r}}=t_{r \bar{r}}=0$, then $Y(N, r)$ solves the dual OMCP, where $r$ is a basic element for which $t_{r g}<0$, and $t_{r j} \leq 0$ for all $j \in N$.

Theorem 4.5 (Basis-Form Duality Theorem) Let $M=\left(\hat{E}_{2 n}, \mathcal{F}\right)$ be an oriented matroid satisfying Assumption 4.3 and the assumptions (3.13) and (3.14) of Theorem 3.1. Then exactly one of the following statements holds:

(a) there exists a complementary feasible basis;

(b) there exists a complementary cofeasible basis.

A basis $B$ or tableau $T(B)$ is called terminal if $B$ is complementary and either feasible or cofeasible. We shall prove the theorem above using the following algorithm, called the criss-cross method, which will be shown to find a terminal tableau after a finite number of steps if the assumptions are satisfied.

In order to describe the algorithm, the following lemma is important.

Lemma 4.6 Let $M=\left(\hat{E}_{2 n}, \mathcal{F}\right)$ be an oriented matroid satisfying the assumptions of Theorem 4.5. Let $B$ be a complementary basis with $g \notin B$. Then, for each $r, s \in B$ the following statements hold:

(1) $t_{r g} \neq 0$ implies $t_{r \bar{r}} \geq 0$;

(2) $t_{r g}=-, t_{r \bar{r}}=t_{s \bar{r}}=0$ and $t_{r \bar{s}}=+i m p l y t_{s g}=-$ and $t_{s \bar{s}}=+$;

(3) $t_{r g}=-, t_{r \bar{r}}=t_{s \bar{s}}=0$ and $t_{r \bar{s}}=+i m p l y t_{s \bar{r}}=-$. 


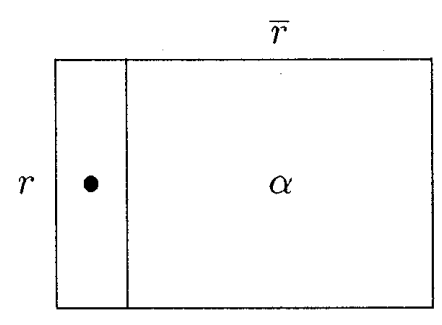

$$
\Longrightarrow \alpha=\oplus
$$

( indicates a nonzero entry)

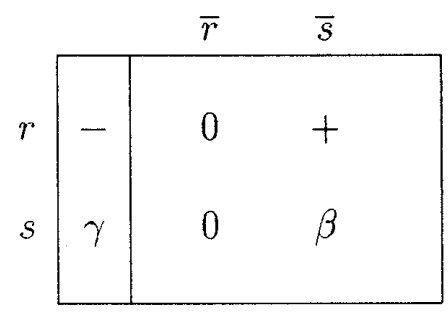

$$
\Longrightarrow \gamma=-, \beta=+
$$

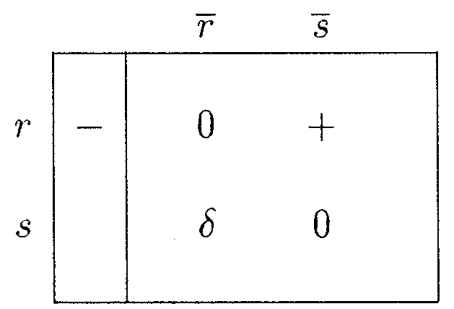

$\Longrightarrow \delta=-$

Figure 4.2: Sign Pattern of Complementary Tableaux

Proof. For the proof, we set $X^{0}=X(B, g), X^{1}=X(B, \bar{r}), X^{2}=X(B, \bar{s}), Y^{1}=Y(N, r)$, and $Y^{2}=Y(N, s)$.

(1) Assume $t_{r g} \neq 0$. Let $t_{r g} \neq 0$ for some $r \in B$, and suppose $t_{r \bar{r}}=-$. Then $X^{1}$ is s.s.r. and $X_{g}^{1}=0$. On the other hand, the vector $Z=\left(t_{r g} X^{0}\right) \circ X^{1} \in \mathcal{F}$ is s.s.p. and $Z_{g} \neq 0$. This contradicts to the assumption (3.13).

(2) Assume $t_{r g}=-, t_{r \bar{r}}=t_{s \bar{r}}=0$ and $t_{r \bar{s}}=+$. Let $\gamma=t_{s g}$ and $\beta=t_{s \bar{s}}$. First we show $\beta=+$. Suppose $\beta \leq 0$. Then the vector $Z=X^{2} \circ\left(-X^{1}\right) \in \mathcal{F}$ is s.s.r. with $Z_{g}=0$, and the vector $W=X^{0} \circ\left(-X^{1}\right) \in \mathcal{F}$ is s.s.p with $W_{g} \neq 0$. This contradicts to the assumption (3.13). Therefore $\beta=+$.

Now, we show $\gamma=-$. Suppose $\gamma \geq 0$. Clearly, the vector $W=Y^{1} \circ\left(-Y^{2}\right) \in \mathcal{F}^{*}$ is s.s.p. with $W_{g} \neq 0$. It suffices to show that there is an s.s.r. vector, say $Z$ in $\mathcal{F}^{*}$ with $Z_{g}=0$, since this contradicts to the assumption (3.14). There are two cases to consider.

Case: $\gamma=0$. In this case, the vector $Z=Y^{2}$ is an s.s.r. vector with $Z_{g}=0$, and hence a contradiction.

Case: $\gamma=+$. In this case, by eliminating the $g$-component from the vectors $Y^{1}$ and $Y^{2}$ using the axiom (OM4), we have a vector $Z \in \mathcal{F}^{*}$, which is s.s.r. with $Z_{g}=0$.

This completes the proof.

(3) Assume $t_{r g}=-, t_{r \bar{r}}=t_{s \bar{s}}=0$ and $t_{r \bar{s}}=+$. Let $\delta=t_{s \bar{r}}$. We have to show $\delta=-$. Suppose $\delta \geq 0$. Then the vector $W=X^{0} \circ\left(-X^{1}\right) \in \mathcal{F}$ is s.s.p. with $W_{g} \neq 0$ and the vector $Z=X^{2} \circ\left(-X^{1}\right)$ is s.s.r. with $Z_{g}=0$. A contradiction to the assumption (3.13).

Figure 4.2 illustrates the meaning of the lemma. 


\section{Criss-Cross method}

The criss-cross method starts with any complementary basis $B$ such that $g \notin B$. The algorithm assumes that the $n$ complementary pairs $\left\{\{e, \bar{e}\}: e \in E_{2 n}\right\}$ are linearly ordered, and this order induces a linear order on any complementary subset of $E_{2 n}$. We denote by $\min S$ the smallest element in $S$, for any complementary subset $S$.

If it terminates then it outputs a pair $(B, e)$ where $B$ is a terminal basis, and $e=g$ if $B$ is feasible, $e=r$ if $B$ is detected cofeasible because of the $r$-row of $T(B)$.

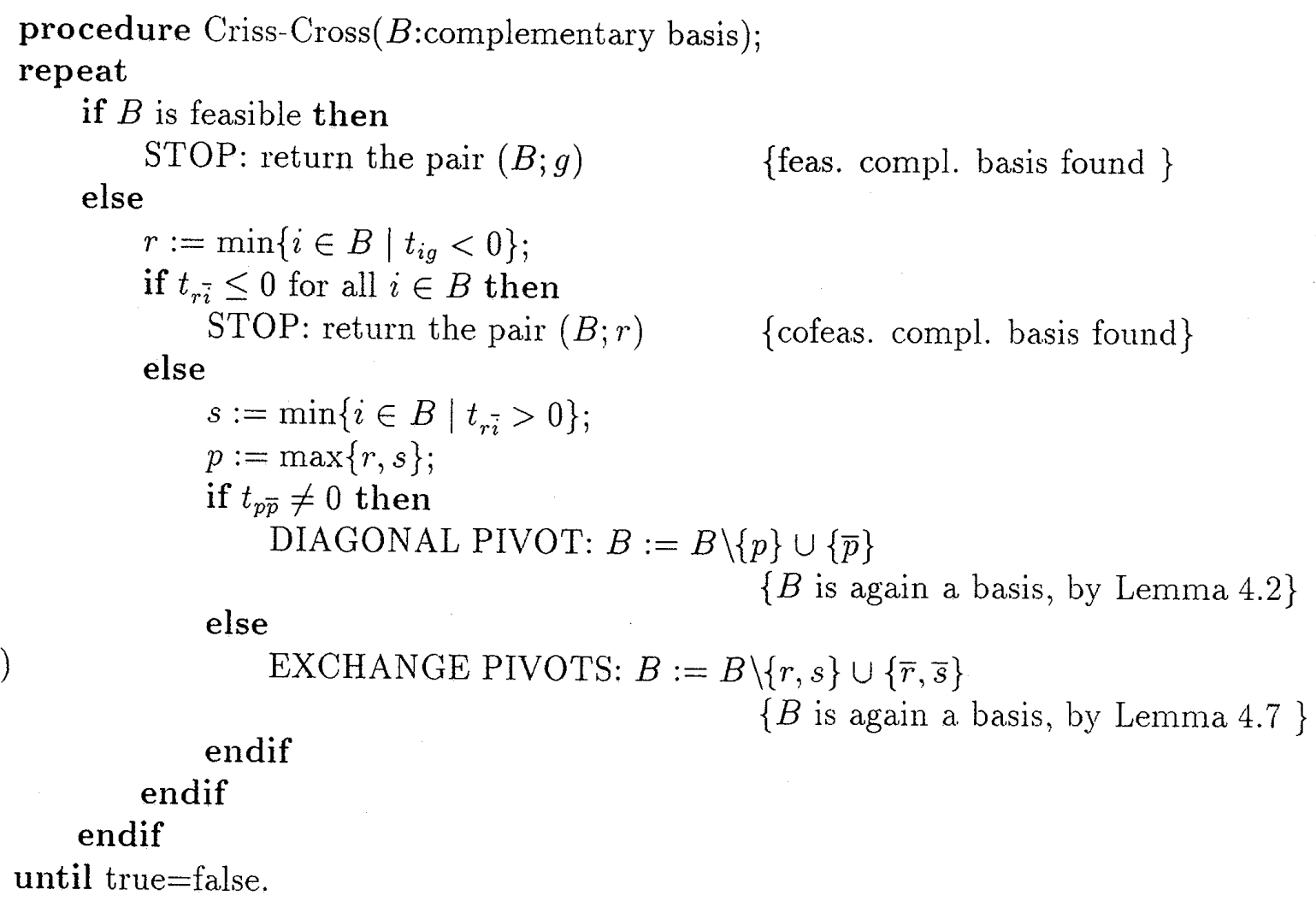

Lemma 4.7 Let $M=\left(\hat{E}_{2 n}, \mathcal{F}\right)$ be an oriented matroid satisfying the conditions (3.13) and (3.14) of Theorem 3.1. When the criss-cross method is applied to $M$, the exchange pivots $\left({ }^{*}\right)$ always produce a complementary basis.

Proof. Let $B$ be a complementary basis produced by the criss-cross method for which the exchange pivots $\left(^{* *}\right)$ are executed. Let $r, s, p$ be indices chosen for $B$ by the algorithm. First consider the case $p=r$. Since $\left(^{* *}\right)$ is executed, $r>s, t_{r g}=-, t_{r \bar{r}}=0$ and $t_{r \bar{s}}=+$. Also, we have $t_{s g} \geq 0$. By Lemma $4.6(2), t_{s \bar{r}} \neq 0$. This together with Lemma 4.2 implies $B \backslash\{r, s\} \cup\{\bar{r}, \bar{s}\}$ is a basis again.

Now consider the case $p=s$. Since ( $\left.{ }^{* *}\right)$ is executed, $s>r, t_{r g}=-, t_{r \bar{s}}=+$ and $t_{s \bar{s}}=0$. Also, by the description of the algorithm and Lemma $4.6(1), t_{r \bar{r}}=0$. By Lemma 4.6 (3), we have $t_{s \bar{r}}=-$. This implies $B \backslash\{r, s\} \cup\{\bar{r}, \bar{s}\}$ is a basis again.

Lemma 4.8 Let $M=\left(\hat{E}_{2 n}, \mathcal{F}\right)$ be an oriented matroid satisfying the conditions (3.13) and (3.14) of Theorem 3.1. Then for each $e \in E_{2 n}$, at most one of the following four statements holds:

(a1) $\exists$ a complementary basis $B^{1}$ not containing $g$ such that $\bar{e} \in B^{1}, t_{i g}^{1} \geq 0$ for all $i \in B^{1}\{\bar{e}\}$ and $t_{\bar{e} g}=-$; 


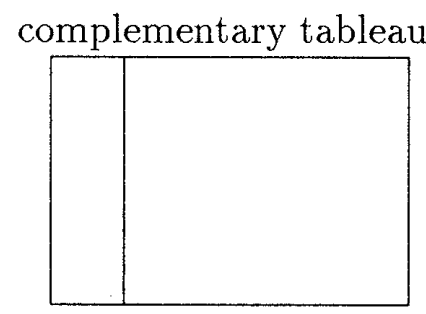

$r: \min ($ top most)
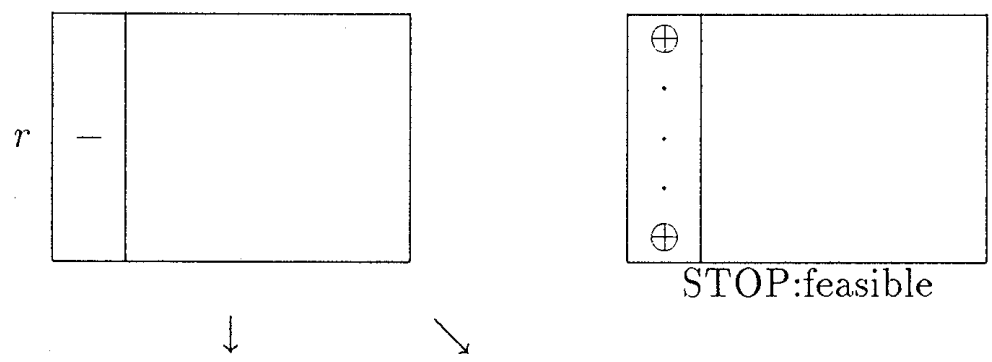

STOP:feasible

$\bar{s}: \min ($ left most)

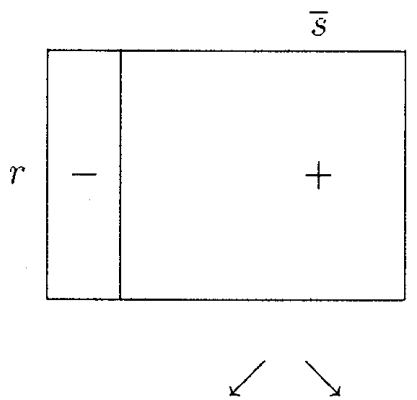

STOP:cofeasible

$$
\begin{aligned}
& p:=\max \{r, s\} \\
& q:=\min \{r, s\}
\end{aligned}
$$

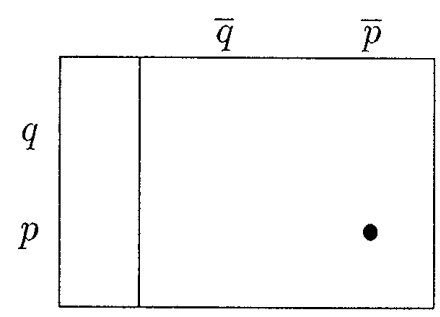

Diagonal Pivot \

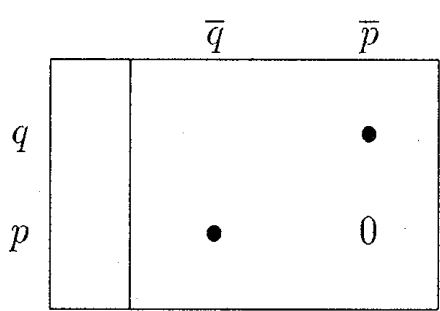

Exchange Pivots

complemantary tableau

Figure 4.3: Criss-Cross Algorithm for the Complementarity Problem 

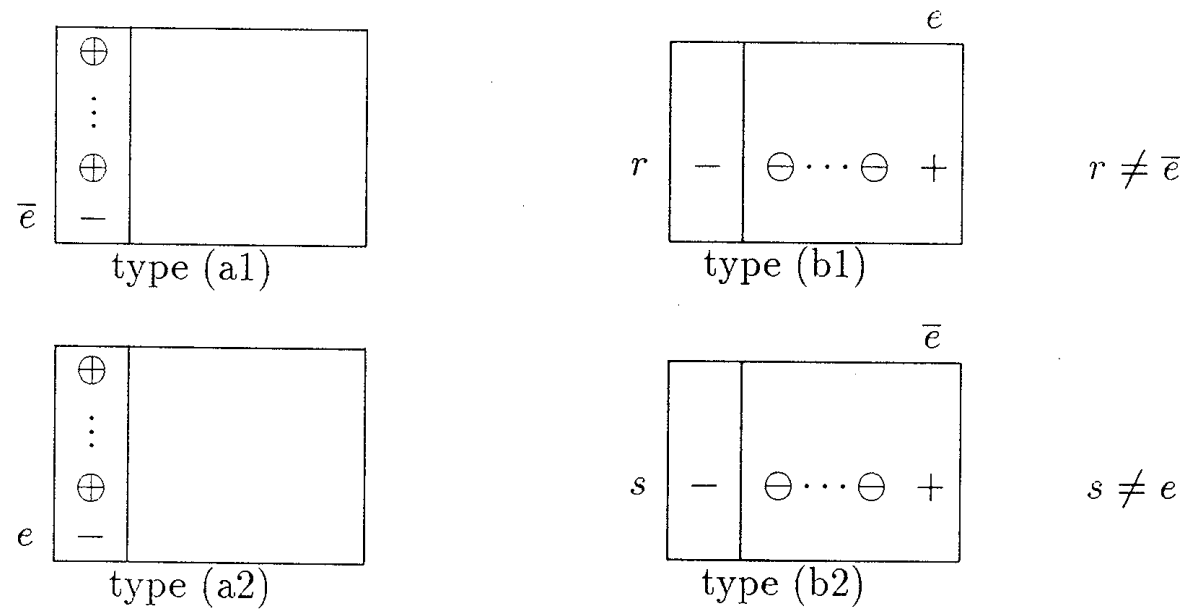

Figure 4.4: Four types of tableaux - Only one type can exist!

(a2) $\exists$ a complementary basis $B^{2}$ not containing $g$ such that $e \in B^{2}, t_{i g}^{2} \geq 0$ for all $i \in B^{2}\{e\}$ and $t_{e g}^{2}=-$;

(b1) $\exists$ a complementary basis $B^{3}$ not containing $g$ such that $\bar{e} \in B^{3}, t_{r g}^{3}<0, t_{r \bar{i}}^{3} \leq 0$ for all $i \in B^{3} \backslash\{\bar{e}\}$ and $t_{r e}^{3}=+$, for some $r \in B^{3}$ with $r \neq \bar{e}$;

(b2) $\exists$ a complementary basis $B^{4}$ not containing $g$ such that $e \in B^{4}, t_{s g}^{4}<0, t_{s \bar{i}}^{4} \leq 0$ for all $i \in B^{4} \backslash\{e\}$ and $t_{s \bar{e}}^{4}=+$, for some $s \in B^{4}$ with $s \neq e$,

where $T\left(B^{k}\right)=\left(t_{i j}^{k}\right)$ for $k=1,2,3,4$.

Proof. Let $X^{i}=X\left(B^{i}, g\right)$ for $i=1,2, Y^{1}=Y\left(N^{3}, r\right)$ and $Y^{2}=Y\left(N^{4}, s\right)$, where $N^{j}=\hat{E}_{2 n} \backslash B^{j}$. By Lemma $4.6(1), t_{r \bar{r}}^{3}=t_{s \bar{s}}^{4}=0$, and therefore the dual vectors $Y^{1}$ and $Y^{2}$ are both complementary. It is easy to see that the vectors $X^{1}, X^{2}, Y^{1}$ and $Y^{2}$ satisfy the conditions in (a1), (a2), (b1) and (b2) of Lemma 3.4, respectively. By Lemma 3.4, at most one of the four vectors exists. Hence, at most one of the four bases $B^{1}, B^{2}, B^{3}, B^{4}$ exists.

The four types of tableaux described in Lemma 4.8 are illustrated in Figure 4.4.

Theorem 4.9 (Finiteness of the Criss-Cross Method) Let $M=\left(\hat{E}_{2 n}, \mathcal{F}\right)$ be an oriented matroid satisfying Assumption 4.3 and the assumptions (3.13) and (3.14) of Theorem 3.1. Then the criss-cross method solves the OMCP associated with $M$, that is, it terminates after a finite number of pivots, and obtains either a complementary feasible basis or a complementary cofeasible basis.

Proof. Suppose the criss-cross method does not terminate for some OMCP problem satisfying the conditions of the theorem, and let $M=\left(\hat{E}_{2 n}, \mathcal{F}\right)$ be such an example with minimal number of elements. Since there are finitely many bases in $M$, it must produce a cycle of adjacent bases: $B^{0}, B^{1}, \ldots, B^{k}=B^{0}$. Because of the minimality of number of elements, within this cycle every element in $E_{2 n}$ must leave and enter some basis. Let $\{e, \bar{e}\}$ be the largest complementary pair in $E_{2 n}$. Consider the situation when enters a basis. By the description of the algorithm, there are two possible cases:

Case E1: $r=\bar{e}$ and $t_{\bar{e} g}$ is the only negative entry in the $g$-column of the tableau;

Case E2: $r \neq \bar{e}, s=\bar{e}$ and $t_{r e}$ is the only positive entry in the $r$-row of the tableau. When $e$ leaves a basis, there are also two possible cases: 
Case L1: $r=e$ and $t_{e g}$ is the only negative entry in the $g$-column of the tableau;

Case L2: $r \neq e, s=e$ and $t_{r \bar{e}}$ is the only positive entry in the $r$-row of the tableau.

These four cases (E1), (E2), (L1), (L2) fit exactly the cases (a1), (b1), (a2), (b2) of Lemma 4.8, respectively. By the same lemma, no two of the four statements can occur, and no two of (E1), (E2), (L1) and (L2) can occur, a contradiction. Therefore, the criss-cross method must terminate. Clearly, when it terminates, the final basis is either a complementary feasible basis or a complementary cofeasible basis.

The duality theorem, Theorem 4.5, is a straightforward consequence of Theorem 4.9.

It is interesting to note that in [16] Grave gave a finite pivot method for LCPs with PSDmatrices, which is somewhat similar to our algorithm. The critical difference is that Grave's algorithm depends on the actual values of tableau entries and it assumes nondegeneracy.

\section{Concluding Remarks}

The criss-cross method we described in the previous section is merely one of finite algorithms for the OMCP (satisfying the conditions of Theorem 4.9). As for the special case of the Oriented Matroid Programming case, one can design different finite pivoting algorithms. In some sense, the criss-cross method is a most rigid algorithm among them, since each pivot operation is uniquely determined and a sequence of complementary bases generated is completely determined once a linear order of complementary pairs $\{e, \bar{e}\}$ is fixed.

One way to see the possibility of relaxing the algorithm is to prove the finiteness of the criss-cross method more constructively as in [14]. In fact, we can show that some binary number associated with each iteration of the algorithm increases monotonically. For each $e \in E_{2 n}$, we denote by $[e]$ the complementary pair $\{e, \bar{e}\}$. Clearly $[e]=[\bar{e}]$. Let $\left[E_{2 n}\right]=$ $\left\{[e] \mid e \in E_{2 n}\right\}$. Thus $\left[E_{2 n}\right]$ is the set $E_{2 n}$ with each complementary pairs $e, \bar{e}$ identified. As it was assumed for the criss-cross method, we assume that the $n$ complementary pairs $\left\{\{e, \bar{e}\}: e \in E_{2 n}\right\}$ are linearly ordered, and this order is just a linear order on $\left[E_{2 n}\right]$. For a $\{0,1\}$-vector $L$ in $\{0,1\}^{\left[E_{2 n}\right]}$, and for $j \in\left[E_{2 n}\right]$, let $L+j$ denote the vector in $\{0,1\}^{\left[E_{2 n}\right]}$

$$
(L+j)_{i}=\left\{\begin{array}{ll}
0 & \text { if } i<j \\
1 & \text { if } i=j \\
L_{i} & \text { if } i>j
\end{array} \quad\left(i \in\left[E_{2 n}\right]\right)\right.
$$

We say $L^{\prime}$ is lex greater than $L$ if $L \neq L^{\prime}$ and $L^{\prime}$ is larger than $L$ in the last subscript $j$ for which they differ. Obviously, if $L_{j}=0$ for some $j$, then $L+j$ is lex greater than $L$.

Let us consider the following updating of $L$ at each iteration of the criss-cross method:

$$
\begin{aligned}
& \text { set } \mathrm{L}=0 \text { initially, and } \\
& \text { update } \mathrm{L} \text { by } \mathrm{L}+\mathrm{p}
\end{aligned}
$$

where $p$ is the larger element $\max \{r, s\}$ between the two basic elements $r$ and $s$ chosen at each iteration.

Theorem 5.1 (Monotonic Improvements) Let $M=\left(\hat{E}_{2 n}, \mathcal{F}\right)$ be an oriented matroid satisfying Assumption 4.3 and the assumptions (3.13) and (3.14) of Theorem 3.1. If the criss-cross method is applied to $M$ with the vector $L$ updated by (5.1), then $L$ strictly increases at each iteration, and therefore the algorithm must terminate in $2^{n}-1$ iterations. 
We omit the proof of this theorem. One just have to show $L_{p}=0$ holds before being updated by $L+p$, and the fundamental lemma Lemma 4.7 is essential again as it was for the proof of Theorem 4.9.

One implication of the theorem is that as long as $L$ increases at each iteration, the algorithm is finite. Consider $L$ at a general stage of the criss-cross method. A zero interval of $L$ is a set of consecutively ordered indices: $j_{1}, j_{2}, \ldots, j_{k} \in\left[E_{2 n}\right]$ such that $L_{j_{1}}=L_{j_{2}}=$ $\ldots=L_{j_{k}}=0$. One can easily see that the linear order among the indices (complementary pairs) is not essential at this stage. So one can pretend that each zero intervals are not ordered, and one can just set any linear ordering on a zero interval whenever necessary.

For the case of linear programming, interesting experimental results supporting the practicality of some flexible version of the criss-cross method have been reported in [22].

\section{References}

[1] Björner, A., Las Vergnas, M., Sturmfels, B., White, N., and Ziegler, G.: Oriented Matroids. Cambridge University Press, to appear.

[2] Bland, R.G. A combinatorial abstraction of linear programming. J. Combin. Theory, Ser. B, Vol. 23 (1977), 33-57.

[3] Bland, R.G., and Las Vergnas, M.: Orientability of matroids. J. Combin. Theory, Ser. $B$, Vol. 24 (1978), 94-123.

[4] Bokowski, J., and Sturmfels, B.: An infinite family of minor-minimal nonrealizablechirotopes. Mathematishe Zeitshrift Vol. 200 (1989), 583-589.

[5] Chung, S.J.: NP-completeness of the linear complementarity problem. J. Optim. Theory Appl., Vol. 60 (1989), 393-399.

[6] Cottle, R.W.: Symmetric dual quadratic programs. Quart. Appl. Math., Vol. 21 (1963), 237-243.

[7] Cottle, R.W.: The principal pivoting method of quadratic programming. in Dantzig, G.B., and Veinott, A.F., (eds.) Mathematics of Decision Sciences, Part 1, American Mathematical Society, Providence, R.I. (1968), 142-162.

[8] Cottle, R.W., and Dantzig, G.B.: Complementary pivot theory of mathematical programming. Linear Algebra and Its Applications, Vol. 1 (1968), 103-125.

[9] Cottle, R.W., Pang, J.-S., and Venkateswaran, V.: Sufficient matrices and the linear complementarity problem. Technical Report SOL 87-15, Stanford University, 1987.

[10] Cottle, R.W.: The principal pivoting method revisited. Technical Report SOL 89-3, Stanford University, 1989.

[11] Den Hertog, D., Roos, C., and Terlaky, T.: The linear complementarity problem, sufficient matrices and the criss-cross method. Reports of The Faculty of Technical Mathematics and Informatics, Delft University of Technology, 1990.

[12] Folkman, J., and Lawrence, J.: Oriented matroids. J. Combin. Theory, Ser. B, Vol. 25 (1978), 199-236. [13] Fukuda, K.: Oriented Matroid Programming. Ph.D. Thesis, University of Waterloo,
1982. 
[14] Fukuda, K., and Matsui, T.: On the finiteness of the criss-cross method. Europ. J. Oper. Res., Vol. 52 (1991), 119-124.

[15] Fukuda, K., and Terlaky, T.: A general recursion algorithm for quadratic programming. preprint, 1989.

[16] Graves, R.L.: A principal pivoting simplex algorithm for linear and quadratic programming. Operations Research, Vol. 15 (1967), 482-494.

[17] Klafszky, E., and Terlaky, T.: Some generalizations of the criss-cross method for quadratic programming. Linear Algebra and Its Applications, to appear.

[18] Klafszky, E., and Terlaky, T.: Some generalizations of the criss-cross method for the linear complementarity problem of oriented matroids. Combinatorica Vol. 9 (1989), 189-198.

[19] Kojima, M., Mizuno, S., and Yoshise, A.: An $O(\sqrt{n} L)$ iteration potential reduction algorithm for linear complementarity problems. Mathematical Programming, Vol. 50 (1991) 331-342.

[20] Lemke, C.E.: Bimatrix equilibrium points and mathematical programming. Management Science, Vol. 11 (1965), 681-689.

[21] Mandel, A.: Topology of Oriented Matroids. Ph.D. Thesis, University of Waterloo, 1982.

[22] Namiki, M., and Matsui, T.: Some modifications of the criss-cross method. Research Report, Department of Information Sciences, Tokyo Institute of Technology, Tokyo, Japan, 1990.

[23] Schrijver, A.: Theory of Linear and Integer Programming. John Wiley 63 Sons, 1986.

[24] Terlaky, T.: A convergent criss-cross method. Mathematische Operationsforschung und Statistics ser. Optimization, Vol. 16 (1985), 683-690.

[25] Terlaky, T.: A finite criss-cross method for oriented matroids. J. of Combin. Theory, Ser. B, Vol. 42 (1987), 319-327.

[26] Todd, M.J.: Complementarity in oriented matroids. SIAM J. Alg. Disc. Meth., Vol. 5 (1984), 467-485.

[27] Todd, M.J.: Linear and quadratic programming in oriented matroids. J. of Combin. Theory, Ser. B, Vol. 39 (1985), 105-133.

[28] Wang, Zh.: A conformal elimination free algorithm for oriented matroid programming. Chinese Annals of Mathematics, 8 B 1 (1987).

[29] Ye, Y., and Pardalos, P.: A class of linear complementarity problems solvable in polynomial time. Department of Management Sciences, The University of Iowa, Iowa. City, Iowa, 1989.

Komei FUKUDA : Graduate School of Systems Management, University of Tsukuba 3-29-1 Otsuka, Bunkyo-ku, Tokyo 112, Japan

Tamás TERLAKY : Faculty of Technical Mathematics and Informatics, Delft University of Technology P.O. Box 35, 2600 AJ Delft, Netherlands 